\title{
Condiciones sanitarias del albergue "COPACO" del bañado norte de Asunción, Paraguay (2018)
}

\author{
Ayelén Mendoza ${ }^{1 D}$, Ulises Mendoza ${ }^{1}$, Esther Pedrozo ${ }^{2}$ iD, Sandra Irala², Guillermo \\ Sequera2 $^{\text {iD }}$, Jorge Canese ${ }^{1}$, Pasionaria Ramos ${ }^{1,2}$ iD \\ ${ }^{1}$ Universidad Nacional de Asunción, Facultad de Ciencias Médicas, Cátedra de Microbiología. \\ Asunción, Paraguay \\ ${ }^{2}$ Ministerio de Salud Pública y Bienestar Social. Dirección General de Vigilancia de la Salud. \\ Asunción, Paraguay
}

Cómo referenciar este artículo/ How to reference this article:
Mendoza A, Mendoza U, Pedrozo E, Irala S, Sequera G, Canese J, Ramos P. Condiciones sanitarias del albergue "COPACO" del bañado norte de Asunción, Paraguay (2018). Mem. Inst. Investig. Cienc. Salud. 2021; 19(1): 31-36

\begin{abstract}
RES U M E N
Los migrantes son más susceptibles a las enfermedades infecciosas, por las malas condiciones sanitarias donde viven. El objetivo fue describir las condiciones sanitarias del albergue "COPACO" del Bañado Norte en el 2018. Estudio descriptivo, transversal y temporalmente prospectivo. Fueron georreferenciados, ubicación y cantidad de grifos, sanitarios, desechos no biológicos, y medidas las distancias entre el agua potable y los desechos. Se evaluó el acceso y calidad del agua potable almacenada y fueron encuestadas las familias para conocer las enfermedades más prevalentes. Las referencias fueron las establecidas en el Manual ESFERA. Fueron contabilizadas 481 familias (2,405 personas); censados 22 baños químicos, 25 grifos, 5 puestos de ducha y 4 puntos de acumulación de desechos. El rango de distancia entre los baños y los 4 grifos más cercanos fue de 1 a $78 \mathrm{~m}$, promedio $28 \mathrm{~m}$. La distancia promedio entre el acúmulo de basura y los 4 grifos más cercanos fue de $21,1 \mathrm{~m}$, rango 1 a $35 \mathrm{~m}$. Cada grifo fue utilizado por 96 personas, 60\% (9) de las familias almacenaban agua. Un baño móvil, con retrete y sin ducha era utilizado por 109 personas. Los baños eran unisex (mixtos) y carecían de fuente de agua y se improvisaron 5 puestos para ducharse, de utilización familiar. Fueron encuestadas 15 familias $(3,1 \%)$. El promedio de estadía 33,3 días, 3 familias acusaron padecer de vómito, fiebre, diarrea y dolor dental. Los resultados pueden orientar a las autoridades sobre las futuras acciones a realizar para salvaguardar la salud de la población migrante
\end{abstract}

Palabras clave: sanitarias, desplazados, albergue, Paraguay.

\section{Sanitary conditions of the "COPACO" quarantine facility in the northern riverside slum of Asuncion, Paraguay (2018)}

\footnotetext{
ABSTRACT

Migrants are more susceptible to infectious diseases, due to the poor sanitary conditions they live in. The objective of this work was to describe the sanitary conditions of the "COPACO" shelter in Bañado Norte (North Slum) in 2018. This was a descriptive, cross-sectional and temporally prospective study. The location and quantity of taps, toilets, non-biological waste were georeferenced, and the distances between drinking water and garbage were measured. Access and quality of stored drinking water were evaluated and families were surveyed to find out the most prevalent diseases. The references were those established in the SPHERE handbook. The number of families counted were 481 (2,405 people), 22 chemical toilets, 25 taps, 
5 shower stalls and 4 garbage accumulation points were registered. The distance range between the toilets and the 4 closest taps was 1 to $78 \mathrm{~m}$, average $28 \mathrm{~m}$. The average distance between the garbage accumulation points and the 4 closest taps was $21.1 \mathrm{~m}$, the range was 1 to $35 \mathrm{~m}$. Each tap was used by 96 people, while 60\% (9) of the families store water. A portable bathroom, with a toilet and without a shower, was used by 109 people. The bathrooms were unisex (mixed) and lacked a water source and 5 stalls were improvised for showering, for family use. Fifteen families were surveyed $(3.1 \%)$. The average stay in the facilities was 33.3 days, and 3 families reported suffering from vomiting, fever, diarrhea and dental pain. The results can guide authorities on future actions to be taken to safeguard the health of the migrant population

Keywords: sanitary, displaced, shelter, Paraguay.

\section{INTRODUCCIÓN}

El desplazamiento forzado o migración forzada se da cuando las personas se ven obligadas a movilizarse de una localidad a otra por situaciones de emergencia tales como accidentes, guerras, fenómenos naturales o cualquier otra situación que ponga en riesgo su supervivencia ${ }^{(1,2)}$. Las personas afectadas por estas crisis son más susceptibles a las enfermedades, principalmente diarreicas e infecciosas. Una de las principales razones por las que se da este fenómeno es por la mala condición sanitaria, es decir, la incorrecta distribución y baja calidad del agua. Otras causas lo constituyen, la falta de organización en cuanto a la ubicación de los sanitarios y el deficiente manejo de los desechos no biológicos ${ }^{(3,4)}$. A estos inconvenientes, se le añade un deficiente manejo del agua dentro de los propios hogares, por lo que en conjunto todos estos factores favorecen la contaminación del agua potable. La Organización Mundial de la Salud (OMS) establece la ausencia de cualquier microrganismo patógeno en el agua, para que pueda ser apta para el consumo, y este parámetro es utilizado como guía para las diversas normativas a nivel nacional e internacional $^{(5,6)}$.

La migración forzada se va tornando cada vez más notoria en la actualidad principalmente por los fenómenos climáticos. Uno de los principales, a nivel mundial, lo constituye la inundación, que posee un componente socio-natural cuando ocurre en zonas habitadas. Este aspecto, es importante de reconocer, puesto que aumenta la vulnerabilidad de las personas ante las precipitaciones leves o intensas. Algunos parámetros a tener en cuenta para la identificación de los posibles riesgos de la población afectada son: conocimiento de la epidemiología de la región, las características sociodemográficas, el acceso a agua potable, el estado nutricional de la población y el esquema de vacunación de las personas ${ }^{(7,8)}$. A su vez, los desastres naturales generan impacto a corto, mediano y largo plazo. A corto plazo, generan exacerbaciones de las patologías crónicas (hipertensión arterial, Diabetes Mellitus, cardiopatías) debido a la falta de acceso a los medicamentos. A mediano plazo, favorece las arbovirosis (dengue) y las enfermedades relacionadas con aguas contaminadas como las leptospirosis. A largo plazo, incrementa la incidencia de depresión en la población afectada debido a la sensación de abandono social. Debido a la gran afectación que puede ocasionar un desastre natural, este puede originar un aumento repentino de las necesidades de una región tales como la necesidad de ambulancias, traslado aéreo, unidades médicas móviles, carpas, espacios de instalación temporal y nueva infraestructura sanitaria. Por la premura de la situación el Estado muchas veces no puede dar una respuesta eficaz ${ }^{(9-11)}$.

A nivel mundial, durante el año 2004 la ciudad de Bangladesh se inundó y posterior a este fenómeno se registró un brote con más de 17.000 casos de enfermedades diarreicas. Del agua que consumían las personas se aislaron $V$. cholerae y $E$. coli enterotoxigénica. En el mismo año, en la provincia de Aceh, Indonesia, luego de un tsunami se comprobó que el $100 \%$ de los sobrevivientes que bebieron agua de contenedores no protegidos desarrollaron una enfermedad diarreica. $Y$, por último, en los Estados Unidos luego de los huracanes Allison y Katrina se notificaron casos de enfermedades diarreicas debido a norovirus, Salmonella spp. y V. cholerae en las 
personas evacuadas de la región afectada ${ }^{(8)}$. A nivel regional, en Brasil se registraron 2 inundaciones importantes, una en el año 2008 en Santa Catarina y otra en el 2010 en Pernambuco, dejando como resultado un aumento de los casos de dengue, así como un deterioro de la infraestructura sanitaria en las regiones afectadas por el desastre natural. La inversión que tuvo que realizar el gobierno brasilero para subsanar esta situación fue de 29,2 millones de reales ${ }^{(9)}$.

Paraguay presenta una alta frecuencia de desastres naturales de índole hídrica, específicamente lluvias torrenciales, inundaciones y tormentas eléctricas ${ }^{(9)}$. En el año 2015 unas 32.402 familias fueron afectadas por las inundaciones, de las cuales 23.736 fueron asistidas por la Secretaria de Emergencia Nacional (SEN) ${ }^{(10)}$. A pesar del gran impacto que generan estos fenómenos naturales al país, las investigaciones sobre este tema son escasas o nulas. El objetivo de este trabajo fue describir las condiciones higiénicas sanitarias del albergue "COPACO" de Bañado Norte, Asunción, Paraguay durante el año 2018.

\section{METODOLOGÍA}

El trabajo se realizó en tres etapas, utilizando la metodología cuantitativa, y el tipo de estudio fue descriptivo, transversal y prospectivo. En la primera parte fueron georreferenciados los indicadores utilizados para evaluar las condiciones sanitarias de la población desplazada. Las variables que fueron medidas fueron: ubicación y cantidad de grifos, ubicación y cantidad de sanitarios, ubicación y manejo de desechos no biológicos, distancia entre la fuente de agua potable y los desechos biológicos y no biológicos. Estos puntos fueron trasladados a un mapa, a partir de los cuales fueron calculadas las distancias existentes entre los grifos, los contenedores y los servicios sanitarios. Para esta etapa no se realizó ningún tipo de muestreo, ya que fueron censados cada uno de los indicadores más arriba mencionados.

En la segunda parte se realizó una evaluación acerca del acceso y calidad del agua potable almacenada en este albergue. El agua potable almacenada debe estar cubierta. Para realizar esta evaluación se confeccionó una lista de verificación (checklist) donde cada una de las variables fue medida por observación directa. Las variables medidas fueron: almacenamiento de agua (si/no), contenedores utilizados (tambores, bidones, baldes) y forma de almacenamiento (abierto/cerrado). El límite para cada variable fue tomado del proyecto ESFERA ${ }^{(3)}$.

La tercera parte de la investigación se realizó con las familias, donde el muestreo fue no probabilístico por conveniencia. Esta tercera etapa tuvo como objetivo conocer las enfermedades más prevalentes en la población vulnerable $(<5$ años, > 60 años, embarazadas y discapacitados). Para ello se confeccionó un breve cuestionario. Los criterios de inclusión fueron todas aquellas personas mayores de edad que aceptaron participar del estudio. Las preguntas fueron realizadas por los investigadores.

La población enfocada fueron todas aquellas familias desplazadas por la inundación y que se encontraban asentadas en un albergue. La población accesible fueron todas aquellas familias desplazadas por las inundaciones que se encontraban asentadas en el albergue "COPACO" durante el año 2018.

En cuanto a las consideraciones éticas se respetaron los principios básicos de la bioética. Entre ellos la beneficencia; ya que estos datos pueden ser utilizados por las autoridades correspondientes para mejorar las condiciones sanitarias de las familias desplazadas. La no maleficencia; no se perjudicó el medio ambiente ni las instalaciones del lugar visitado; la autonomía, en cuanto a que fue respetada la decisión de las personas en caso de no querer participar de las encuestas realizadas y la justicia; no fue excluida ninguna persona por razones de credo, sexo, raza, edad, preferencias políticas ni cualquier otro motivo similar.

\section{RESULTADOS}

El asentamiento contó con 481 familias (2.405 personas). Fueron censados 22 baños químicos, 25 grifos, 5 puestos de ducha y 4 puntos de acumulación de desechos. El rango de distancia entre los baños y los 4 grifos más cercanos fue de 1 a $78 \mathrm{~m}$, promedio $28 \mathrm{~m}$. y la distancia promedio entre el acúmulo de basura y los 4 
grifos más cercanos fue de $21,1 \mathrm{~m}$, rango 1 a $35 \mathrm{~m}$. Cada grifo era utilizado por 96 personas, $60 \%{ }^{(9)}$ de las familias almacenaban agua. Un baño móvil, con retrete y sin ducha era utilizado por 109 personas. Los baños eran unisex y carecían de fuente de agua. Se identificaron 5 puestos improvisados para ducharse, de utilización familiar (Figura 1).

Fuente: Mendoza A., Mendoza U., Pedrozo E., Irala S., Sequera G., Canese J., Ramos P. Condiciones Sanitarias del albergue "COPACO" de Bañado Norte de Asunción, Paraguay (2018).

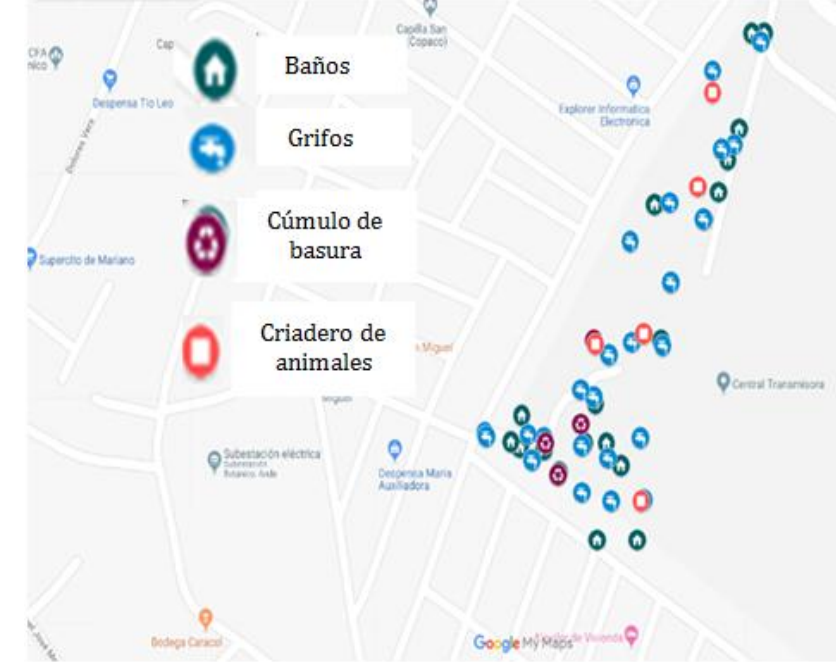

Figura 1: Distribución de los grifos, baños y disposición de basuras en el albergue "COPACO" del Bañado Norte de Asunción, Paraguay (2018)

Fueron encuestadas 15 familias (3,1 \% del total). El promedio de estadía fue de 33,3 días, 3 familias acusaron padecer de vómito, fiebre, diarrea y dolor dental durante su estadía.

En materia de abastecimiento de agua se halló que por cada grifo existen aproximadamente 96 personas que lo utilizan, a su vez, el $60 \%$ (9) de las familias encuestadas presentaban almacenamiento de agua (Tabla 1 ).

Tabla 1: Distribución del almacenamiento del agua de las familias del albergue "COPACO" de Bañado Norte, Asunción, Paraguay (2018).

\begin{tabular}{llll}
\hline Contenedor & Abierto & Cerrado & Total \\
\hline Bidones & 2 & 2 & 4 \\
Tambores & 2 & 2 & 4 \\
Balde & 2 & 1 & 3 \\
\hline
\end{tabular}

Fuente: Mendoza A., Mendoza U., Pedrozo E., Irala S., Sequera G., Canese J., Ramos P. Condiciones Sanitarias del albergue "COPACO" de Bañado Norte de Asunción, Paraguay (2018).

Tabla 2: Relación de los grifos, baños y disposición de basuras en el albergue "COPACO" con relación a los estándares internacionales (2018)

\begin{tabular}{|c|c|c|c|}
\hline VARIABLES & \multicolumn{2}{|c|}{ Albergue "COPACO" } & $\begin{array}{l}\text { Proyecto } \\
\text { ESFERA }\end{array}$ \\
\hline \multicolumn{4}{|l|}{ Distancia } \\
\hline Baño - grifo & $27,8 \mathrm{~m}$ & 1 a $78 \mathrm{~m}$ & $\geq 30 \mathrm{~m}$ \\
\hline $\begin{array}{l}\text { Baño - basura } \\
\text { Relación }\end{array}$ & $21,1 \mathrm{~m}$ & 1 a $35 \mathrm{~m}$ & \\
\hline Persona/grifo & 96 & & \\
\hline Personas/baño & 109 & & 20 por 1 \\
\hline Mujeres/varones por baño & - & - & 3 por 1 \\
\hline
\end{tabular}

En tanto que en el manejo de la evacuación de los excrementos se observó que se cuenta con un baño móvil, con retrete y sin ducha, por cada 109 personas, los baños 
fueron unisex y carecían de fuente de agua. Se identificaron también 5 puestos improvisados para ducharse, de utilización familiar.

Por otra parte, en el ámbito del manejo de los residuos no biológicos no se pudieron divisar contenedores de basura comunitarios, pero se pudo establecer 4 puntos principales de aglomeración de basura.

Por último, para el levantamiento de datos acerca de las enfermedades prevalentes en la comunidad se encuestaron 15 familias, que representan el 3,1 \% del total de las familias asentadas en el albergue, con promedio de estadía de 33,3 días, estas familias representan un total de 66 personas, de las cuales un 30,3\% forman parte de grupos de riesgo; 3 familias acusaron padecer de vómito, fiebre, diarrea y dolor dental durante su estadía.

\section{DISCUSIÓN}

En el país son frecuentes los casos de inundaciones, en general por la crecida cíclica del Río Paraguay, estas traen consigo desplazamientos forzados para las más de 7500 familias que representan un aproximado de 37.500 personas habitantes de las zonas ribereñas; donde las familias son reubicadas en 108 asentamientos a lo largo de la capital del país ${ }^{(8-10)}$. El asentamiento de COPACO constituye uno de los de mayor envergadura de la ciudad, siendo este uno de los criterios tomados para su selección. Los parámetros se encuentran establecidos en el Manual Esfera, para la respuesta humanitaria que son de aceptación mundial ${ }^{(3)}$.

La cantidad de grifos por persona fue adecuada obteniéndose una relación de 1 grifo para 96 personas con lo que se aseguró el suministro de agua potable, sin embargo, no todas las familias poseían contenedores de agua (40\%); de las que lo tenían, no todos se encontraban tapados. Asimismo, no se realizaba la distinción de los contenedores para acarrear el agua y para el consumo del mismo. Estos resultados coinciden con el estudio realizado por Venegas $C$ et al. en el que se observó que, si bien las personas tenían recipientes para el almacenamiento del agua, los mismos no cumplían con los requisitos sanitarios ${ }^{(5)}$.

Los baños con los que contaban eran acondicionados cada día por empresas contratadas por el gobierno, no obstante, estos carecían de una fuente interna de agua para el aseo de las manos. A su vez, la relación baño por persona fue de 1/109, la cual se encuentra muy lejos de cumplir con las recomendaciones internacionales. Otro punto importante, fue que en estos baños no se hacían distinción de uso para los sexos, con lo se genera una mayor vulnerabilidad para las mujeres y niñas del lugar y no se da una intimidad apropiada a las mismas.

La disposición de la basura no era ordenada, las familias no contaban con un contenedor común o comunitario, los desechos se encontraban esparcidos a lo largo del territorio del asentamiento y se desconoce si tienen un sistema de transporte de los mismos. En el asentamiento se pudo identificar 4 puntos de gran acumulación de basura, principalmente reciclable, con fines lucrativos, pero sin ninguna medida de seguridad física o biológica tanto para el reciclador como para las familias asentadas en ese lugar.

La distancia entre la fuente de agua potable y las instalaciones destinadas para realizar las necesidades fisiológicas fue de $27,8 \mathrm{~m}$, la cual es cercana a lo recomendado por el Manual ESFERA $(\geq 30 \mathrm{~m})$. Sin embargo, se pudo observar en algunos casos, que algunas se encontraban sensiblemente próximas. A su vez, la distancia entre los grifos y los desechos no biológicos fue de $21,1 \mathrm{~m}$ por lo que se encontraban bastante próximas, lo que constituye un verdadero riesgo por la posibilidad de contaminación del agua.

Finalmente, el $20 \%$ de las familias encuestadas acusaron padecer algún tipo de patología de tipo aguda desde su instalación en el albergue. Estas cifras son inferiores a la reportada en la población colombiana en situación de desplazamiento forzado siendo la principal causa la afección de las vías aéreas ${ }^{(5)}$. Esta diferencia, puede deberse al tiempo en que fue realizada la investigación.

Esperamos que los resultados de este estudio puedan orientar a las autoridades sobre las acciones futuras a tomar en este tipo de fenómenos climáticos para poder reducir al mínimo los riesgos para la salud de la población migrante. 
Las condiciones sanitarias del albergue "COPACO" no cumplen con las recomendaciones dictadas por las normas internacionales. Las inundaciones constituyen un problema sistemático en el país, por lo que sería recomendable cuidar estos aspectos a fin de evitar la propagación de enfermedades.

\section{REFERENCIAS BIBLIOGRAFÍCAS}

1. Organización Panamericana de la Salud. El agua en situaciones de emergencia. 2010; 32. [citado 2021 Mar 15] Disponible en: https://www.paho.org/es/documentos/ag ua-situaciones-emergencia.

2. Ruiz R, NY. El desplazamiento forzado en Colombia: una revisión histórica y demográfica. Estudios Demográficos y Urbanos, vol. 26, núm. 1, enero-abril, 2011, pp. 141-177

3. Sphere Association. The Sphere Handbook: Humanitarian Charter and Minimum Standards in Humanitarian Response, fourth edition, Geneva, Switzerland, 2018. [citado 2021 Mar 15]. Disponible en: https://spherestandards.org/wpcontent/uploads/Sphere-Handbook-2018EN.pdf

4. Natuzzi ES, Joshua C, Shortus M, Reubin $R$, Dalipanda $T$, Ferran $K$, et al. Defining Population Health Vulnerability Following an Extreme Weather Event in an Urban Pacific Island Environment: Honiara, Solomon Islands. Am J Trop. Med Hyg 2016;95(2):307-14.

5. Venegas C, Mercado M, Campos C. Evaluación de la calidad microbiológica del agua para consumo y del agua residual en una población de Bogotá (Colombia). Revista Biosalud 2014; 13(2): 24-35.

6. Organización Mundial de la Salud. Guías para la Calidad del Agua Potable de la Organización Mundial de la Salud (OMS). Recomendaciones. 3th Edition; 2006. p. 398. [citado 2021 Mar 15]. Disponible en:

https://www.who.int/water_sanitation_he alth/dwq/gdwq3rev/es/

7. Herrera E, Magaña V, Morett S. Relación entre eventos extremos de precipitación con inundaciones. Estudio de caso: Tulancingo, Hidalgo. Nova scientia [revista en la Internet]. 2018 [citado 2021 Mar 15] 10(21):191-206.Disponible en:

http://www.scielo.org.mx/scielo.php?scri pt $=$ sci_arttext\&pid $=$ S2007070520180002 $00191 \bar{l}$ Ing $=$ es. $10.21640 / n s . v 10 i 21.1527$.

8. Watson JT, Gayer M, Connolly MA. Epidemics after Natural Disasters. Emerg Infect Dis. 2007;13(1):1-5.

9. Londe $L$ de $R$, Marchezini $V$, Conceição RS da, Bortoletto KC, Silva AEP, Santos EV dos, et al. Impactos de desastres

socioambientais em saúde pública: estudos dos casos dos Estados de Santa Catarina em 2008 e Pernambuco em 2010. Revista Brasileira de Estudos de População. 16 de diciembre de 2015;32(3):537-62.

10. Salazar-Arbelaez G. Terremotos y salud: lecciones y recomendaciones. Salud Publica Mex 2018; 60 (suppl 1): S6-S15. 10.21149/9445

11. Mora-González AP, Madrid-González DA, Barragán-Vázquez S. 19S: la respuesta en salud del gobierno del estado de Morelos. Salud Publica Mex 2018; 60 (supl 1): S97-S104. 10.21149/9294

12. UNISDR. Asistencia sanitaria en emergencias y desastres- ASANED Paraguay. 2014. [citado 2021 Mar 15]. Disponible en: https://www.eird.org/pr14/sesiones/Or ganizaciones/ASANED-ParaguayAsistencia-Sanitaria-en-Emergencias-yDesastres.pdf

13. SEN. Inundaciones en Asunción y el interior del país. Asunción; 2015. [citado 2021 Mar 15]. Disponible en: https://reliefweb.int/report/paraguay/re porte-de-situaci-n-n-22015-

inundaciones-en-asunci-n-inundacioness-bitas-y

14. Administración Nacional de Navegación y Puertos. Informe Hidrometereológico del Río Paraguay. 2018. [citado 2021 Mar 15]. Disponible en: https://reliefweb.int/report/paraguay/in forme-hidrometeorol-gico-del-r-oparaguay-07-05-2018

15. Última Hora. Suman 7.000 las familias afectadas por las inundaciones en Asunción [Internet]. ultimahora.com. 2018 [citado 28 noviembre 2018]. Disponible en: https://www.ultimahora.com/suman7000-las-familias-afectadas-lasinundaciones-asuncion

16. Paraguay Fluvial. El río Paraguay está por debajo de los niveles críticos y se espera un comportamiento cíclico natural de bajante [Internet]. Paraguay Fluvial. 2018 [citado 28 noviembre 2018]. Disponible en: http://paraguayfluvial.com/el-rioparaguay-esta-por-debajo-de-losniveles-criticos-y-se-espera-uncomportamiento-ciclico-natural-debajante/ 